\title{
Increasing incidence and antimicrobial resistance in Escherichia coli bloodstream infections: a multinational population-based cohort study
}

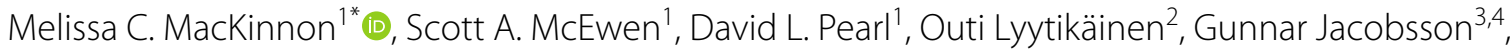 \\ Peter Collignon ${ }^{5,6}$, Daniel B. Gregson ${ }^{7,8}$, Louis Valiquette ${ }^{9}$ and Kevin B. Laupland ${ }^{10,11,12}$
}

\begin{abstract}
Background: Escherichia coli is an important pathogen in humans and is the most common cause of bacterial bloodstream infections (BSIs). The objectives of our study were to determine factors associated with E. coli BSI incidence rate and third-generation cephalosporin resistance in a multinational population-based cohort.

Methods: We included all incident E. coli BSIs (2014-2018) from national (Finland) and regional (Australia [Canberra], Sweden [Skaraborg], and Canada [Calgary, Sherbrooke, and western interior]) surveillance. Incidence rates were directly age and sex standardized to the European Union 28-country 2018 population. Multivariable negative binomial and logistic regression models estimated factors significantly associated with E. coli BSI incidence rate and thirdgeneration cephalosporin resistance, respectively. The explanatory variables considered for inclusion in both models were year (2014-2018), region (six areas), age (<70-years-old and $\geq 70$-years-old), and sex (female and male).

Results: We identified 31,889 E. coli BSIs from 40.7 million person-years of surveillance. Overall and third-generation cephalosporin-resistant standardized rates were 87.1 and 6.6 cases/100,000 person-years, respectively, and increased $14.0 \%$ and $40.1 \%$ over the five-year study. Overall, $7.8 \%$ (2483/31889) of E. coli BSIs were third-generation cephalosporin-resistant. Calgary, Canberra, Sherbrooke, and western interior had significantly lower E. coli BSI rates compared to Finland. The significant association between age and E. coli BSI rate varied with sex. Calgary, Canberra, and western interior had significantly greater odds of third-generation cephalosporin-resistant E. coli BSIs compared to Finland. Compared to 2014, the odds of third-generation cephalosporin-resistant E. coli BSIs were significantly increased in 2016, 2017, and 2018. The significant association between age and the odds of having a third-generation cephalosporin-resistant E. coli BSI varied with sex.
\end{abstract}

Conclusions: Increases in overall and third-generation cephalosporin-resistant standardized E. coli BSI rates were clinically important. Overall, E. coli BSI incidence rates were $40-104 \%$ greater than previous investigations from the same study areas. Region, sex, and age are important variables when analyzing E. coli BSI rates and third-generation cephalosporin resistance in E. coli BSIs. Considering E. coli is the most common cause of BSIs, this increasing burden

*Correspondence: mmacki05@uoguelph.ca;

melissa.c.mackinnon@gmail.com

${ }^{1}$ Department of Population Medicine, University of Guelph, 50 Stone Rd

E, Guelph, ON N1G 2W1, Canada

Full list of author information is available at the end of the article

(c) The Author(s) 2021. Open Access This article is licensed under a Creative Commons Attribution 4.0 International License, which permits use, sharing, adaptation, distribution and reproduction in any medium or format, as long as you give appropriate credit to the original author(s) and the source, provide a link to the Creative Commons licence, and indicate if changes were made. The images or other third party material in this article are included in the article's Creative Commons licence, unless indicated otherwise in a credit line to the material. If material is not included in the article's Creative Commons licence and your intended use is not permitted by statutory regulation or exceeds the permitted use, you will need to obtain permission directly from the copyright holder. To view a copy of this licence, visit http://creativecommons.org/licenses/by/4.0/. The Creative Commons Public Domain Dedication waiver (http://creativeco mmons.org/publicdomain/zero/1.0/) applies to the data made available in this article, unless otherwise stated in a credit line to the data. 
and evolving third-generation cephalosporin resistance will have an important impact on human health, especially in aging populations.

Keywords: Population-based, Bloodstream infection, Bacteremia, Escherichia coli, Incidence rate, Antimicrobial resistance, Third-generation cephalosporins

\section{Background}

Escherichia coli is the most common cause of bloodstream infections (BSIs) (1-3). Regional populationbased studies conducted in Canberra (Australia; 2000-2004), Calgary (Canada; 2000-2006), Funen County (Denmark; 2000-2008), Auckland (New Zealand; 2005-2011), and Skaraborg County (Sweden; 2011-2012, only community-onset $E$. coli BSIs) described annual $E$. coli BSI rates of $28.0-70.2 / 100,000$ population $(2,4-7)$. A national population-based study from England revealed annual rates of $60.4 / 100,000$ population $(04 / 2012$ $03 / 2013)$ and $63.5 / 100,000$ population (04/201303/2014) (8). Recently, some areas worldwide reported an increase in E. coli BSI incidence (8-10). The emergence of third-generation cephalosporin-resistant (3GC-R) $E$. coli has also had a major effect on the epidemiology and treatment of these infections worldwide (11). Thirdgeneration cephalosporin-resistant $E$. coli infections are often multidrug-resistant, and in contrast to many other multidrug-resistant bacteria, they mostly emerge in community settings $(5,6,8,12)$.

There is an increasingly large body of literature on $E$. coli BSIs. However, most studies are hospital-based and typically recruit patients from highly selected populations at large tertiary-care centres. In order to determine the incidence rate of E. coli BSIs and understand the associated burden of disease, a population-based study is required (13). By using this approach, the population at risk can be defined, and selection bias is minimized by including all $E$. coli BSIs from the population (13). To our knowledge, a multinational population-based study evaluating incidence rates and antimicrobial resistance (AMR) in E. coli BSIs has not been previously published.

Using multinational population-based data, we aimed to: 1) evaluate the incidence rate of $E$. coli BSIs and associated factors; and 2) evaluate factors associated with having a 3GC-R E. coli BSI.

\section{Methods}

\section{Study protocol}

For this population-based cohort study, we enrolled six participating surveillance areas from the International Bacteremia Surveillance Collaborative based on their willingness to voluntarily participate in the study, and their ability to provide the required data and meet project timelines (14). Finland contributed active national surveillance data. Regional surveillance data were available from areas within Canada (three areas), Australia (one area), and Sweden (one area). From 01/01/2014 to $31 / 12 / 2018$, all incident episodes of $E$. coli BSIs from residents within the surveillance areas were included, whether they occurred in a hospital or community setting. An incident BSI was defined as growth of $E$. coli from at least one blood culture, and only the first $E$. coli isolate per patient per running year was included (i.e., at least one year of time elapsed between $E$. coli BSIs). The surveillance databases from each area identify an estimated $99 \%$ or greater of all positive blood cultures in their residents, except for Canberra Region, where at least $95 \%$ are detected (as a private laboratory processes some blood cultures) $(14,15)$. We retrieved the following data from electronic medical records: year of culture; patient's sex and age category ( $<1$-year then deciles until $\geq 90$-years); location of onset (hospital-onset or community-onset); and susceptibility to 3GC, ciprofloxacin, gentamicin, trimethoprim/sulfamethoxazole (TMS), and meropenem. Resistance to $3 \mathrm{GC}$ was defined according to each area's established methodology and details are available in Additional file 1. Each area performed susceptibility testing according to their own established protocols. Data for location of onset were not available from Canberra. Susceptibility data for ciprofloxacin, gentamicin, TMS, and meropenem were not available from Canberra and Finland. If the first positive blood culture was obtained at least $48 \mathrm{~h}$ after hospital admission or within $48 \mathrm{~h}$ of hospital discharge, the BSI was characterized as hospital-onset; otherwise, it was characterized as community-onset (16). Individual surveillance areas compiled data using standardized data summary templates. The following research ethics boards approved the study and granted waivers of informed consent: the Interior Health Research Ethics Board (2013-14-052-I); the University of Guelph Research Ethics Board (201810-050); the Conjoint Health Research Ethics Board of the University of Calgary (REB19-1025); the Regional Ethics Board Gothenburg (539-11); the Ethics Committee of the Finnish Institute for Health and Welfare (THL/1349/6.02.00/2019); the ACT (Australian Capital Territory) Health Human Research Ethics Committee (2020.LRE.00115); and Comité d'éthique de la recherche du CIUSSS de l'Estrie-CHUS (Centre intégré universitaire de santé et de services sociaux de l'Estrie-Centre 
hospitalier universitaire de Sherbrooke) (2011-286, 10-181). Analyses related to mortality in E. coli BSIs in this study are documented in a separate manuscript.

\section{Surveillance populations}

The study surveillance populations (2018) included: Calgary Health Region, Canada (1.7 million); Canberra Region, Australia (421,000); country of Finland (5.5 million); Sherbrooke Region, Canada, (166,000); Skaraborg County Health Region, Sweden $(267,000)$; and western interior area of British Columbia, Canada $(191,000)$. Detailed descriptions of each area's population and surveillance methodology have been previously published $(14,15,17)$. Of note, since the time of previous publications, Skaraborg now has two hospitals instead of four.

\section{Data management and statistical analysis}

The data analyses were performed in Stata 15.1 (18). We calculated prevalence for dichotomous variables (sex, location of onset, and AMR data) and to summarize age, we determined the age category that contained the median of the E. coli BSI age distribution. Using univariable logistic regression, an odds ratio (OR) was estimated to compare the odds of a BSI being hospital-onset in 3GC-R and 3GC-susceptible (3GC-S) E. coli BSIs. We calculated incidence rates by dividing the number of incident E. coli BSIs by the surveillance population, which was obtained from individual area census data. To facilitate comparison of incidence rates between different regions and different years, we directly age and sex standardized the incidence rates to the European Union 28-country (EU28) 2018 population $(18,19)$. Incidence rates for 3GC-R and 3GC-S E. coli BSIs were calculated and directly standardized as above. We used a negative binomial regression model to determine factors significantly associated with $E$. coli BSI incidence rates $(18,20)$. To explore factors significantly associated with having 3GC-R E. coli BSIs, we used a logistic regression model $(18,20)$. For inclusion in each regression model, we considered the following four categorical explanatory variables: year (2014 through 2018); region (six study areas); age ( $<70$-years-old and $\geq 70$-years-old); and sex (female and male). Age was dichotomized using a 70-year-old cut-off based on the structure of our data (collected in 10-year age brackets for adults) and to facilitate modelling the risk factor of elderly age. We performed univariable analysis and assessed for high correlation between explanatory variables using a Phi coefficient $(\rho \geq|0.8|$ represented significant correlation) prior to all explanatory variables being placed in the multivariable regression models. We considered interaction effects between year and region, and age and sex for inclusion in the multivariable regression models due to their epidemiologic and / or biologic plausibility. To remain in the final multivariable model, a variable had to be statistically significant $(\alpha=0.05)$, part of a significant interaction term, or an important confounder (based on meeting causal criteria and $>20 \%$ change in another variable's coefficient) (20). The final multivariable negative binomial regression model was assessed for goodness-of-fit (deviance goodness-of-fit test and normality of Anscombe residuals), and residuals (Pearson and deviance), leverage and an influence statistic (Cook's distance) were assessed (20). The final multivariable logistic regression model was assessed for goodness-of-fit (Pearson goodness-offit test), and standardized Pearson residuals, leverage and influence statistics (delta-beta, delta-chi ${ }^{2}$, and deltadeviance) were assessed (20). We performed contrasts to explore interaction terms included in the final multivariable models. Incidence rate ratios (IRR) and OR were reported with $95 \%$ confidence intervals $(\mathrm{CI})$.

\section{Missing data}

We used a casewise deletion method to manage missing data, where incident $E$. coli BSIs were removed from specific analyses if data were incomplete. A total of $17 E$. coli BSIs were removed from the main descriptive and regression modelling analyses due to missing data for age, sex, and third-generation cephalosporin susceptibility. Location of onset was missing for 983 incident $E$. coli BSIs. Data regarding susceptibility to ciprofloxacin, gentamicin, TMS, and meropenem were missing for 25,622, 25,614, 25,606 and 25,739 incident E. coli BSIs, respectively.

\section{Results}

We identified 31,889 incident E. coli BSIs from 40.7 million patient-years of follow-up during the five-year study; $59.1 \%(18,856 / 31,889)$ of those were in females. The median age range for $E$. coli BSI patients was 70-79-years-old, and the distribution was left-skewed (Fig. 1). Overall, 7.8\% (2483/31889) of E. coli BSIs were 3GC-R ranging from $6.1 \%(1510 / 24296)$ in Finland to $17.8 \%(671 / 3773)$ in Calgary. Most E. coli BSIs were community-onset $(82.2 \% ; 25,417 / 30,923)$, and this was lowest in Finland $(80.8 \%, 19,909 / 24,629)$ and highest in Skaraborg $(92.1 \%, 1241 / 1347)$. A 3GC-R E. coli BSI was at greater odds of being hospital-onset compared to 3GC-S (OR:1.68, 95\%CI:1.53-1.85, $p<0.001$ ). The proportion of $E$. coli BSIs that were resistant to ciprofloxacin, gentamicin, TMS, and meropenem was $23.4 \%$ (1473/6284), $11.2 \%$ (702/6292), 26.1\% (1642/6300), and 0.1\% (6/6167), respectively. Additional file 2 contains regional data for AMR and location of onset.

The overall crude E. coli BSI rate was 78.4 cases/100,000 person-years (see Additional file 3). The 


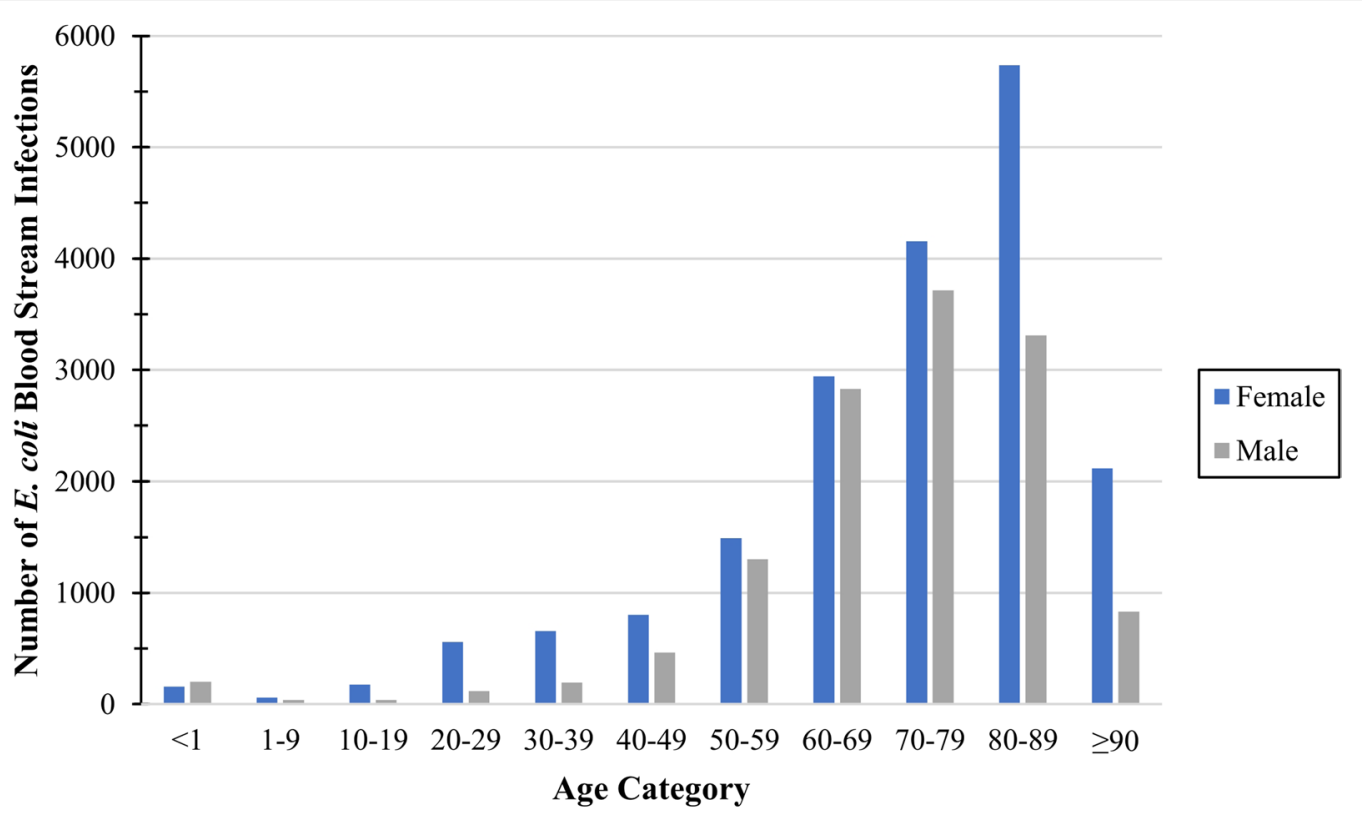

Fig. 1 Number of E. coli bloodstream infections by age category and sex

overall annual directly age and sex standardized rate was 87.1 E. coli BSI/100,000 population, which was lowest in western interior and highest in Skaraborg (64.2 and 93.6 cases/100,000 population) (Fig. 2a). For 3GC-R E. coli BSIs, the overall annual standardized rate was 6.6 cases/100,000 population ranging from 5.0 cases/100,000 population in Sherbrooke to 12.2 cases/100,000 population in Calgary (Fig. 2a, b). From 2014 to 2018, the overall and 3GC-R annual standardized rates increased by $14.0 \%$ and $41.1 \%$, respectively (Fig. 3a, b). Additional file 4 contains regional and annual standardized overall, 3GC-R, and 3GC-S E. coli BSI rates.

With univariable negative binomial regression analysis, there was a significant association between age and E. coli BSI rate, but no variation by year, region, or sex (see Additional file 5). Our multivariable negative binomial model for E. coli BSI rates included region, and an interaction between age and sex (Table 1). Calgary, Canberra, Sherbrooke, and western interior had significantly lower rates of E. coli BSI compared to Finland (Table 1). In terms of the interaction between sex and age, males or females that were $\geq 70$-years-old had significantly higher E. coli BSI rates compared to $<70$-year-old males or females (Table 2). However, while females $<70$-yearsold had significantly higher $E$. coli $\mathrm{BSI}$ rates compared to males $<70$-years-old, there was no significant difference in rates between females and males that were $\geq 70$-yearsold (Table 2).

Region, year, age, and sex were significantly associated with having $3 \mathrm{GC}-\mathrm{R} E$. coli BSIs using univariable logistic regression analysis (see Additional file 6). Our multivariable logistic model for having 3GC-R E. coli BSIs included region, year, and an interaction between age and sex (Table 3). Calgary, Canberra, and western interior had significantly greater odds of 3GC-R E. coli BSIs than Finland (Table 3). Compared to 2014, the odds of 3GC-R E. coli BSIs were significantly increased in 2016, 2017, and 2018 (Table 3). In terms of the interaction between sex and age, males in either age category were at significantly increased odds of having 3GC-R E. coli BSIs compared to females in either age category (Table 4). However, while males $<70$-years-old were at significantly increased odds of having $3 \mathrm{GC}-\mathrm{R}$ E. coli BSIs compared to males $\geq 70$-years-old, there was no significant difference between females in different age categories (Table 4).

\section{Discussion}

Our study provides several notable contributions to the E. coli BSI literature. It is the first multinational population-based study of $E$. coli BSIs, which included all incident cases over a five-year period from six areas in four countries on three continents. The population-based design of the study allowed us to thoroughly capture both community-onset and hospital-onset $E$. coli BSIs; a very important aspect for $E$. coli BSIs since the vast majority are community-onset, including $82.2 \%$ in our study. The five-year timeframe of the study and multinational design allowed us to explore annual and regional variation in the incidence rate and $3 \mathrm{GC}$ resistance of $E$. coli BSIs. We incorporated the demographic factors of age 


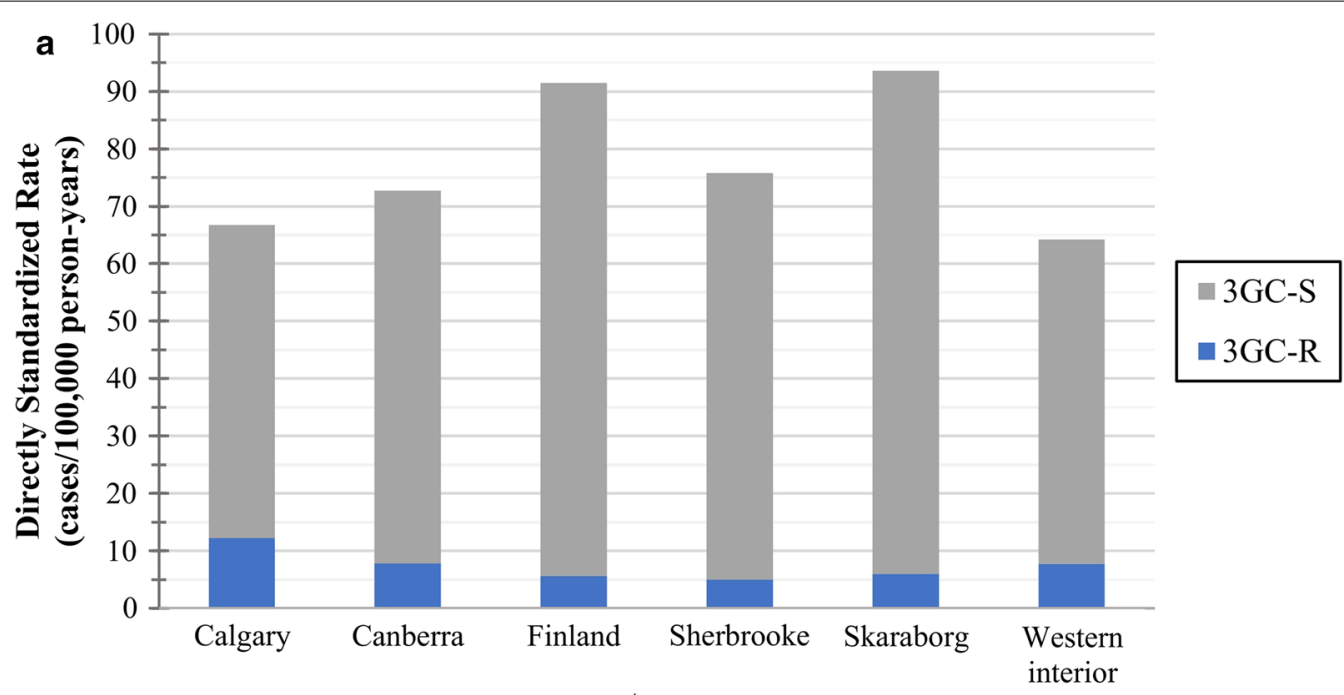

Area

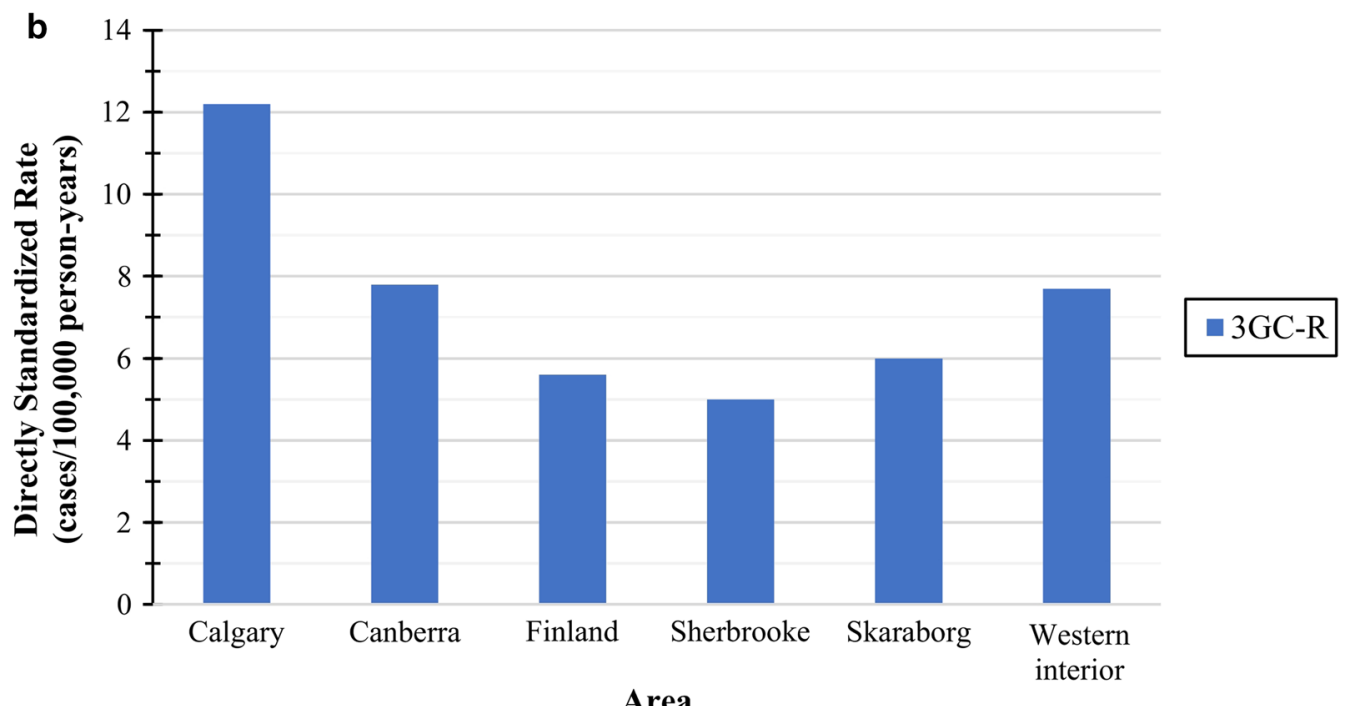

Area

Fig. 2 Directly age and sex standardized E. coli bloodstream infection incidence rates by area,b. a Overall, and third-generation cephalosporin-resistant and -susceptible standardized incidence rates by area ${ }^{\mathrm{a}, \mathrm{b}}$. $\mathbf{b}$ Third-generation cephalosporin-resistant standardized incidence rates by area ${ }^{a, b}$. 3GC-R Third-generation cephalosporin-resistant; 3GC-S Third-generation cephalosporin-susceptible. ${ }^{\text {a }}$ Standard population-

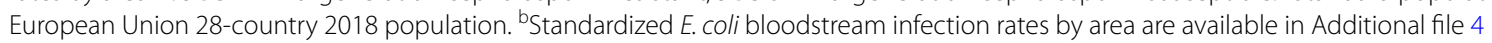

and sex using several techniques, including: direct age and sex standardization of $E$. coli BSI rates to facilitate comparisons of rates between different regions and different years, and with future studies; and inclusion of age and sex in our regression models.

In population-based studies, the incidence rate of infection provides insight into the burden of the disease being studied (13). The overall crude and directly age and sex standardized rates of $E$. coli BSIs in our study were 78.4 and 87.1 cases/100,000 person-years, respectively, and included 31,889 incident E. coli BSIs from 2014 to 2018. We found a higher standardized BSI rate than rates from all previously published population-based studies (Table 5). Our crude BSI rate was also higher than all crude rates (Table 5). We need to note, however, that these rate comparisons are general in nature and should be interpreted cautiously. These rates are from different time periods, and there are differences in demographics between the populations that are unaccounted for when crude rates or rates standardized with a different standard population are compared. Previous population-based studies reported prevalences of 3GC-R E. coli BSIs that varied from $1-10.4 \%(5,6,8,21)$, which is similar to our overall prevalence of $7.8 \%$. 

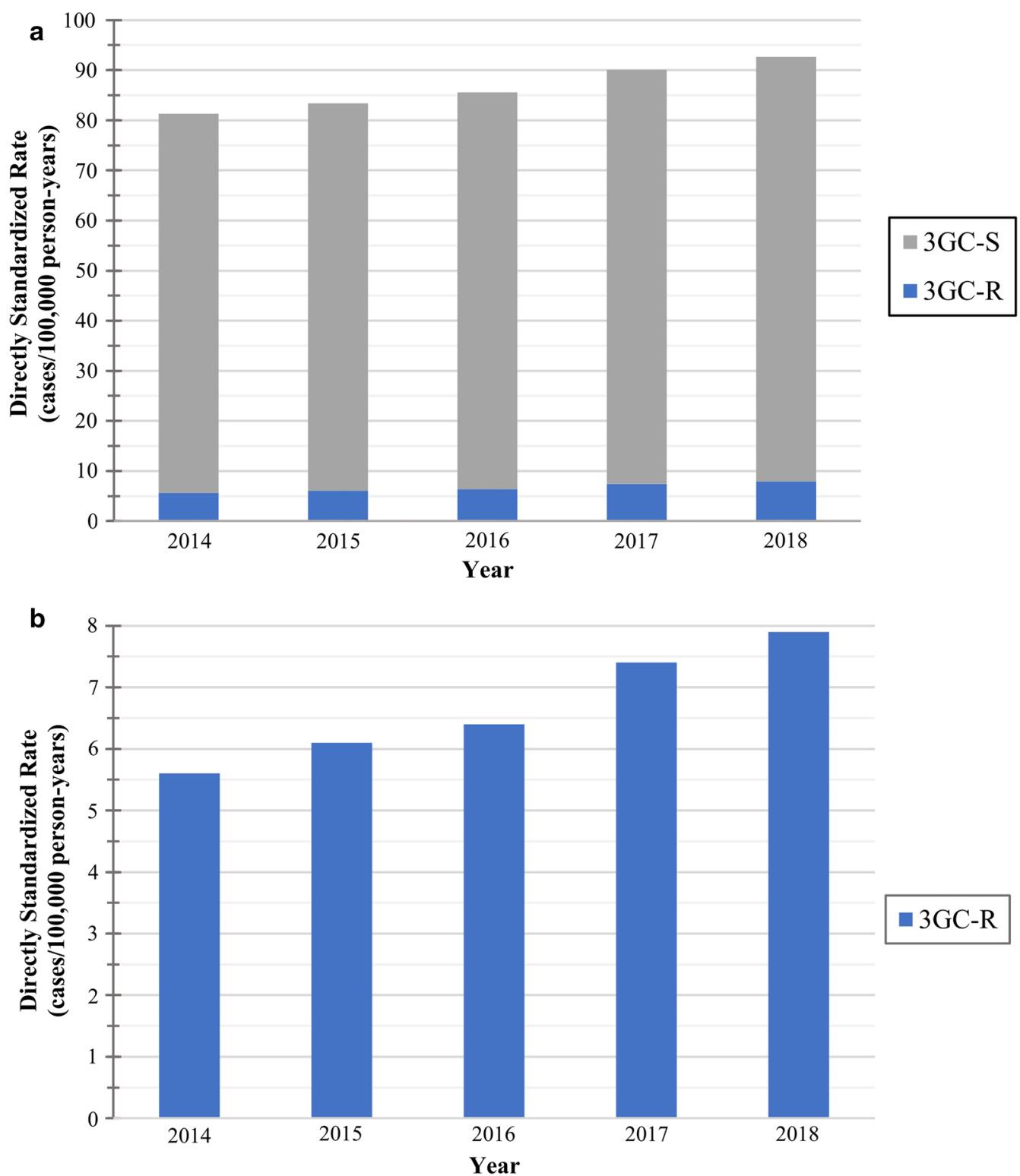

Fig. 3 Directly age and sex standardized E. coli bloodstream infection incidence rates by year ${ }^{\mathrm{a}, \mathrm{b}}$. a Overall, and third-generation cephalosporin-resistant and -susceptible standardized incidence rates by year ${ }^{\mathrm{a}, \mathrm{b}}$. $\mathbf{b}$ Third-generation cephalosporin-resistant standardized incidence rates by year, ${ }^{\mathrm{a}, \mathrm{b}}$. 3GC-R Third-generation cephalosporin-resistant; 3GC-S Third-generation cephalosporin-susceptible. ${ }^{2}$ Standard population-

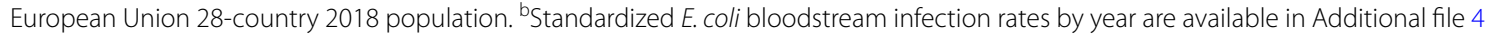

Both the E. coli BSI rate and $3 \mathrm{GC}$ resistance had significant regional differences. The Scandinavian areas had higher E. coli BSI rates but lower odds of 3GC-R E. coli BSIs. The opposite was seen in Canberra, Calgary and western interior. Region likely serves as a proxy variable for many unmeasured regional and population characteristics. Therefore, our study is not able to determine the underlying reason(s) for these regional differences, which also could be different on a national or regional scale. In theory, some regional differences could be due to varying degrees of capturing positive $E$. coli blood cultures by different areas (surveillance system coverage), but this is unlikely with our enrolled surveillance areas because they all captured at least $95 \%$ of all positive blood cultures in their residents. To explore the underlying reason(s) for the regional differences, future studies could evaluate culturing rate, surveillance system coverage, healthcare 
Table 1 Multivariable negative binomial regression model results estimating associations between explanatory variables and E. coli BSI rates ${ }^{\mathrm{a}, \mathrm{b}}$

\begin{tabular}{|c|c|c|c|}
\hline Variable & alRR & $95 \% \mathrm{Cl}$ & $p$-value \\
\hline \multicolumn{3}{|l|}{ Region } & \multirow[t]{2}{*}{$<0.001$} \\
\hline Finland & 1.00 & Referent & \\
\hline Calgary & 0.71 & $0.65-0.77$ & $<0.001$ \\
\hline Canberra & 0.71 & $0.65-0.79$ & $<0.001$ \\
\hline Sherbrooke & 0.86 & $0.77-0.96$ & $<0.001$ \\
\hline Skaraborg & 1.05 & $0.96-1.15$ & 0.312 \\
\hline Western interior & 0.73 & $0.65-0.82$ & $<0.001$ \\
\hline \multicolumn{4}{|l|}{ Sex } \\
\hline Female & 1.00 & Referent & \multirow[b]{2}{*}{$<0.001$} \\
\hline Male & $0.74^{c}$ & $0.67-0.80$ & \\
\hline \multicolumn{4}{|l|}{ Age category } \\
\hline$<70$-years-old & 1.00 & Referent & \\
\hline$\geq 70$-years-old & $9.37^{c}$ & $8.63-10.19$ & $<0.001$ \\
\hline \multicolumn{4}{|c|}{ Interaction—sex and age } \\
\hline Male and $\geq 70$ & $1.39^{c}$ & $1.23-1.57$ & $<0.001$ \\
\hline \multicolumn{4}{|c|}{$\begin{array}{l}\text { BSI Bloodstream infection; alRR Adjusted incidence rate ratio; CI Confidence } \\
\text { interval }\end{array}$} \\
\hline \multicolumn{4}{|c|}{ a Overdispersion parameter $0.014,95 \%$ Cl:0.008-0.025, $p<0.001$} \\
\hline \multicolumn{4}{|c|}{$\begin{array}{l}{ }^{\mathrm{b}} \text { Model fit the data based on normally distributed Anscombe residuals and non- } \\
\text { significant deviance goodness-of-fit test }(p=0.24)\end{array}$} \\
\hline \multicolumn{4}{|c|}{$\begin{array}{l}\text { ' Exponentiated coefficients are not true aOR due to interaction term-see } \\
\text { contrasts in Table } 2\end{array}$} \\
\hline
\end{tabular}

Table 2 Results for contrasts examining interactions between sex and age based on multivariable negative binomial regression model $^{\mathrm{a}}$

\begin{tabular}{lrcr}
\hline Contrast statement & IRR & 95\% Cl & p-value \\
\hline Females $\geq 70$ compared to females $<70$ & 9.37 & $8.63-10.19$ & $<0.001$ \\
Males $\geq 70$ compared to females $<70$ & 9.61 & $8.83-10.45$ & $<0.001$ \\
Females $\geq 70$ compared to males $<70$ & 12.72 & $11.68-13.86$ & $<0.001$ \\
Males $\geq 70$ compared to males $<70$ & 13.04 & $11.96-14.22$ & $<0.001$ \\
Females $\geq 70$ compared to males $\geq 70$ & 0.98 & $0.90-1.06$ & 0.558 \\
Females $<70$ compared to males $<70$ & 1.36 & $1.24-1.48$ & $<0.001$
\end{tabular}

IRR Incidence rate ratio; $\mathrm{Cl}$ Confidence interval

${ }^{\text {a }}$ Multivariable negative binomial regression model estimating the associations between the explanatory variables (region, sex, and age) and E. coli bloodstream infection incidence rate (Table 1)

practices (including antimicrobial use), ethnicity, socioeconomic status, and climate, among other factors.

Over the 5-year study period, we identified a clinically important $14.0 \%$ increase in the overall standardized $E$. coli BSI rate but did not find significant annual variation in overall $E$. coli BSI rate with negative binomial regression analysis. Previous investigations from areas enrolled in the current study reported overall crude rates of 28 , 30.3, 44, 67 cases/100,000 person-years for Canberra
Table 3 Multivariable logistic regression model results estimating associations between explanatory variables and having 3GC-R E. coli BSI ${ }^{a}$

\begin{tabular}{|c|c|c|c|}
\hline Variable & $\mathrm{aOR}$ & $95 \% \mathrm{Cl}$ & $p$-value \\
\hline \multicolumn{3}{|l|}{ Region } & \multirow[t]{2}{*}{$<0.001$} \\
\hline Finland & 1.00 & Referent & \\
\hline Calgary & 3.25 & $2.94-3.59$ & $<0.001$ \\
\hline Canberra & 1.87 & $1.52-2.31$ & $<0.001$ \\
\hline Sherbrooke & 1.04 & $0.75-1.45$ & 0.796 \\
\hline Skaraborg & 1.02 & $0.81-1.27$ & 0.875 \\
\hline Western interior & 2.08 & $1.61-2.70$ & $<0.001$ \\
\hline \multicolumn{3}{|l|}{ Year } & \multirow[t]{2}{*}{$<0.001$} \\
\hline 2014 & 1.00 & Referent & \\
\hline 2015 & 1.07 & $0.92-1.23$ & 0.388 \\
\hline 2016 & 1.15 & $1.00-1.33$ & 0.048 \\
\hline 2017 & 1.23 & $1.07-1.41$ & 0.003 \\
\hline 2018 & 1.31 & $1.15-1.50$ & $<0.001$ \\
\hline \multicolumn{4}{|l|}{ Sex } \\
\hline Female & 1.00 & Referent & \multirow[b]{2}{*}{$<0.001$} \\
\hline Male & $1.83^{b}$ & $1.61-2.09$ & \\
\hline \multicolumn{4}{|l|}{ Age category } \\
\hline$<70$-years-old & 1.00 & Referent & \\
\hline$\geq 70$-years-old & $1.05^{\mathrm{b}}$ & $0.93-1.19$ & 0.420 \\
\hline \multicolumn{4}{|c|}{ Interaction — sex and age } \\
\hline Male and $\geq 70$ & $0.84^{b}$ & $0.71-0.999$ & 0.048 \\
\hline \multicolumn{4}{|c|}{$\begin{array}{l}3 G C-R \text { Third-generation cephalosporin-resistant; } B S I \text { Bloodstream infection; } a O R \\
\text { Adjusted odds ratio; } C I \text { Confidence interval }\end{array}$} \\
\hline \multicolumn{4}{|c|}{$\begin{array}{l}{ }^{a} \text { Model fit the data based on non-significant Pearson goodness-of-fit test } \\
(p=0.072)\end{array}$} \\
\hline
\end{tabular}

Table 4 Results for contrasts examining interactions between sex and age based on multivariable logistic regression model ${ }^{a}$

\begin{tabular}{lccr}
\hline Contrast statement & OR & $\mathbf{9 5 \% ~ C l}$ & $\boldsymbol{p}$-value \\
\hline Males $<70$ compared to females $<70$ & 1.83 & $1.61-2.09$ & $<0.001$ \\
Males $\geq 70$ compared to females $<70$ & 1.62 & $1.44-1.84$ & $<0.001$ \\
Females $\geq 70$ compared to females $<70$ & 1.05 & $0.93-1.19$ & 0.420 \\
Males $<70$ compared to females $\geq 70$ & 1.74 & $1.55-1.96$ & $<0.001$ \\
Males $\geq 70$ compared to females $\geq 70$ & 1.54 & $1.39-1.72$ & $<0.001$ \\
Males $<70$ compared to males $\geq 70$ & 1.13 & $1.00-1.27$ & 0.045 \\
\hline
\end{tabular}

OR Odds ratio; $\mathrm{Cl}$ Confidence interval

${ }^{a}$ Multivariable logistic regression model estimating the associations between the explanatory variables (region, year, sex, and age) and having a thirdgeneration cephalosporin-resistant E. coli bloodstream infection (Table 3)

(2000-2004), Calgary (2000-2006), Finland (2004-2007), Skaraborg (2011-2012, community-onset only), respectively $(1,4,5,7)$. The rates reported in our study represent notable rate increases between each of the study 
Table 5 Study details and E. coli bloodstream infection incidence rates from previously published population-based studies

\begin{tabular}{|c|c|c|c|c|}
\hline Study location & Study dates & $\begin{array}{l}\text { Number of } E \text {. coli } \\
\text { BSI }\end{array}$ & $\begin{array}{l}\text { Incidence rate (cases/100,000 } \\
\text { person-years) }\end{array}$ & Type of incidence rate \\
\hline Olmsted County, USA (21) & 1998-2007 & 461 & 41.4 & Standardized ${ }^{\mathrm{a}}$ \\
\hline Canberra, Australia (4) & $2000-2004$ & 515 & 28 & Crude \\
\hline \multirow[t]{3}{*}{ Funen County, Denmark (2) } & $2000-2002$ & 811 & 70.2 & Crude \\
\hline & $2003-2005$ & 718 & 61.8 & Crude \\
\hline & 2006-2008 & 671 & 56.7 & Crude \\
\hline \multirow[t]{2}{*}{ mid-Norway (9) } & 2002-2013 & 686 & 80 & Crude \\
\hline & 2002-2013 & 686 & 74 & Standardized ${ }^{b}$ \\
\hline Calgary, Canada (5) & 2000-2006 & 2,368 & 30.3 & Crude \\
\hline Finland (national) (1) & 2004-2007 & 9,190 & 44 & Crude \\
\hline Auckland, New Zealand (6) & $2006-2011$ & 1,507 & 52 & Crude \\
\hline Skaraborg County, Sweden (7) & 2011-2012 & $104^{c}$ & 67.0 & Crude \\
\hline \multirow[t]{2}{*}{ Two rural Thai provinces (10) } & 2008 & 373 & 32.9 & Crude \\
\hline & 2014 & 593 & 51.6 & Crude \\
\hline \multirow[t]{2}{*}{ England (national) (8) } & 04/2012-03/2013 & 32,309 & 60.4 & Crude \\
\hline & 04/2013-03/2014 & 34,203 & 63.5 & Crude \\
\hline
\end{tabular}

$B S /$ Bloodstream infection

${ }^{\text {a }}$ USA 2000 white population standard

${ }^{\mathrm{b}}$ Norway 2010 population

c Only community-onset E. coli BSI with severe sepsis

pairs of $71 \%, 52 \%, 104 \%$, $40 \%$ in Canberra, Calgary, Finland, and Skaraborg, respectively. It appears that the rate of increase was likely even higher prior to the 2014 beginning of our study. We found a considerable (41.1\%) increase in the 3GC-R E. coli BSI rate over our 5-year study period and an increase in the odds of having $3 \mathrm{GC}-\mathrm{R}$ E. coli BSIs in 2016-2018 compared to 2014. The association between year and having 3GC-R E. coli BSIs did not depend on the region being considered, since the interaction term between year and region was not statistically significant. This increase in the proportion of 3GC-R E. coli BSIs will have important impacts on patient burden, healthcare burden and empirical antimicrobial therapy guidelines since 3GC-R and extended-spectrum beta-lactamase-producing E. coli BSIs have been associated with increased treatment failure, mortality, length of hospital stay, and hospital costs (22-25).

Age and sex are demographic factors that are known to impact the rate and odds of disease and may do so through interaction, such that the relationship depends on which combination of sex and age are being considered $(8,15)$. Our multivariable negative binomial regression model for E. coli BSI rate and multivariable logistic regression model for having 3GC-R E. coli BSIs both included a significant interaction term between age and sex. We did not identify a previous population-based study that explored interaction between age and sex, however, previous studies have shown that females and older patients had an increased rate of $E$. coli BSIs $(1,3$, $5,9,26)$. This reinforces the importance of considering interaction terms between age and sex for inclusion in multivariable models.

There are some limitations to this study. We did not have information to further categorize the communityonset BSIs into community-acquired and healthcareacquired. Culturing rates were not available for the enrolled areas (27). We did not have access to information regarding comorbidities, source of $E$. coli BSI, or treatment. The laboratory methodology was based on each area's protocol, and therefore, there may have been some small methodological differences. Population data were provided by each area and although they all have highly developed procedures for census and population estimation, there are likely some methodological differences between areas. All of the areas were from highincome countries limiting the generalizability of our results to comparable countries (28). There is one E. coli BSI population-based study from two rural provinces in Thailand, an upper-middle-income country (10); however, more population-based research on E. coli BSIs in low, lower-middle, and upper-middle-income countries is needed to understand the global burden of disease (28). 


\section{Conclusions}

Our multinational population-based study demonstrated the substantial and increasing burden of E. coli BSIs. During the 5-year study, there was a clinically important increase in the overall and 3GC-R standardized $E$. coli BSI rates and a noteworthy significant increase in the odds of having 3GC-R E. coli BSIs in 2016-2018. There were significant regional, sex, and age differences in both the rate and 3GC resistance of E. coli BSIs. These findings highlight the difficulty in comparing and extrapolating results from single-centre studies where there are likely differences in unmeasured regional factors and demographics. Considering $E$. coli is the most common cause of BSIs, this increasing burden and evolving 3GC resistance will have an important impact on human health, especially in aging populations.

\section{Abbreviations}

3GC: Third-generation cephalosporin; 3GC-R: Third-generation cephalosporinresistant; 3GC-S: Third-generation cephalosporin-susceptible; AMR: Antimicrobial resistance; BSI: Bloodstream infection; BSIs: Bloodstream infections; E. coli: Escherichia coli; EU28: European Union 28-country; IRR: Incidence rate ratio; OR: Odds ratio; TMS: Trimethoprim/sulfamethoxazole.

\section{Supplementary Information}

The online version contains supplementary material available at https://doi. org/10.1186/s13756-021-00999-4.

Additional file 1. Table summarizing the methodology used to determine susceptibility to third-generation cephalosporins by the enrolled areas.

Additional file 2. Table containing proportion of E. coli bloodstream infections by region that were resistant to third-generation cephalosporins, ciprofloxacin, gentamicin, trimethoprim/sulfamethoxazole, or meropenem and location of onset.

Additional file 3. Table containing counts of incident E. coli bloodstream infections, length of patient follow-up and crude rates of E. coli bloodstream infections.

Additional file 4. Table containing directly age and sex standardized E. coli bloodstream infection incidence for overall, third-generation cephalosporin-resistant and susceptible E. coli bloodstream infections

Additional file 5. Table containing the crude incidence rate ratios for the univariable negative binomial regression models estimating associations between E. coli bloodstream infection rates, and region, year, sex and age.

Additional file 6 . Table containing the crude odds ratios for the univariable logistic regression models estimating associations between having a third-generation cephalosporin-resistant E. coli bloodstream infection, and region, year, sex and age.

\section{Acknowledgements}

GJ would like to thank Niklas Klaar and Ulrika Eriksson for help with data collection. LV would like to thank Anaïs Marcil-Héguy and Cynthia Grenier for help with data collection. We thank members of the International Bacteremia Surveillance Collaborative for support of this project: Kevin B. Laupland (Kamloops, Canada); Daniel B. Gregson, Deirdre L. Church (Calgary, Canada); Louis Valiquette (Sherbrooke, Canada); Outi Lyytikainen (Helsinki, Finland); Peter Collignon, Karina J. Kennedy (Canberra, Australia); Gunnar Jacobsson (Skaraborg, Sweden); John Galbraith, Pamela Kibsey, Kennard Tan (Victoria, Canada); Henrik C. Schonheyder (Aalborg, Denmark); Jenny Dahl Knudsen, Jens Otto
Jarlov, Christian Ostergaard Anderson, Mette Pinholt (Copenhagen, Denmark); Kim Oren Gradel (Odense, Denmark); and Frank Brunkhorst (Jena, Germany).

\section{Authors' contributions}

MCM: contributed to data collection; conducted the data analyses; and drafted and edited the manuscript; KBL, OL, GJ, PC, DBG, and LV: contributed to data collection; and manuscript preparation; SAM and DLP: contributed to data analyses; and manuscript preparation. All authors read and approved the final version of the manuscript.

\section{Funding}

No external funding was received as direct support for this project. MCM's PhD program is supported by a Banting and Charles Best Canadian Graduate Scholarship Doctoral Award from the Canadian Institutes of Health Research, a Brock Doctoral Scholarship from the University of Guelph, an OVC Scholarship from the Ontario Veterinary College at the University of Guelph and funding from the Genome Research Development Initiative through the Government of Canada. The funding organizations were not involved in any stage of the project.

\section{Availability of data and materials}

The aggregated datasets analyzed during the current study may be available from the corresponding author on reasonable request.

\section{Declarations}

\section{Ethics approval and consent to participate}

The following research ethics boards approved the study and granted waivers of informed consent: the Interior Health Research Ethics Board (2013-14-052-I); the University of Guelph Research Ethics Board (2018-10-050); the Conjoint Health Research Ethics Board of the University of Calgary (REB19-1025); the Regional Ethics Board Gothenburg (539-11); the Ethics Committee of the Finnish Institute for Health and Welfare (THL/1349/6.02.00/2019); the ACT (Australian Capital Territory) Health Human Research Ethics Committee (2020. LRE.00115); and Comité d'éthique de la recherche du CIUSSS de I'EstrieCHUS (Centre intégré universitaire de santé et de services sociaux de l'EstrieCentre hospitalier universitaire de Sherbrooke) (2011-286, 10-181)

\section{Consent for publication}

Not applicable.

\section{Competing interests}

MCM reports a scholarship from the Canadian Institutes of Health Research and funding for a Federal Student Work Experience Program placement from the Government of Canada_-Genome Research Development Initiative, both related to her PhD program and outside the submitted work. LV reports being a stock holder of Lumed Inc., outside the submitted work. All other authors declare that they have no competing interests.

\section{Author details}

${ }^{1}$ Department of Population Medicine, University of Guelph, 50 Stone Rd E, Guelph, ON N1G 2W1, Canada. ${ }^{2}$ Department of Health Security, National Institute for Health and Welfare, Helsinki, Finland. ${ }^{3}$ Department of Infectious Diseases, Skaraborg Hospital, Skövde, Sweden. ${ }^{4}$ CARe - Center for Antibiotic Resistance Research, Institute of Biomedicine, University of Gothenburg, Gothenburg, Sweden. ${ }^{5}$ Department of Infectious Disease and Microbiology, The Canberra Hospital, Garran, ACT, Australia. ${ }^{6}$ Medical School, Australian National University, Acton, ACT, Australia. ${ }^{7}$ Departments of Medicine, and Pathology and Laboratory Medicine, University of Calgary, Calgary, AB, Canada. ${ }^{8}$ Alberta Health Services, Calgary Zone, Calgary, AB, Canada. ${ }^{9}$ Department of Microbiology-Infectious Diseases, Université de Sherbrooke, Sherbrooke, QC, Canada. ${ }^{10}$ Department of Medicine, Royal Inland Hospital, Kamloops, BC, Canada. ${ }^{11}$ Department of Intensive Care Medicine, Royal Brisbane and Women's Hospital, Brisbane, QLD, Australia. ${ }^{12}$ Faculty of Health, Queensland University of Technology (QUT), Brisbane, QLD, Australia.

Received: 2 November 2020 Accepted: 23 August 2021

Published online: 06 September 2021 


\section{References}

1. Skogberg K, Lyytikainen O, Ollgren J, Nuorti JP, Ruutu P. Populationbased burden of bloodstream infections in Finland. Clin Microbiol Infect. 2012;18(6):E170-6.

2. Nielsen SL, Pedersen C, Jensen TG, Gradel KO, Kolmos HJ, Lassen AT Decreasing incidence rates of bacteremia: a 9-year population-based study. J Infect. 2014;69(1):51-9.

3. Bonten M, Johnson JR, van den Biggelaar AHJ, Georgalis L, Geurtsen J, de Palacios PI, et al. Epidemiology of Escherichia coli bacteremia: a systematic literature review. Clin Infect Dis. 2020;72(7):1211-9.

4. Kennedy KJ, Roberts JL, Collignon PJ. Escherichia coli bacteraemia in Canberra: incidence and clinical features. Med J Aust. 2008;188(4):209-13.

5. Laupland KB, Gregson DB, Church DL, Ross T, Pitout JDD. Incidence, risk factors and outcomes of Escherichia coli bloodstream infections in a large Canadian region. Clin Microbiol Infect. 2008;14(11):1041-7.

6. Williamson DA, Lim A, Wiles S, Roberts SA, Freeman JT. Population-based incidence and comparative demographics of community-associated and healthcare-associated Escherichia coli bloodstream infection in Auckland, New Zealand, 2005-2011. BMC Infect Dis. 2013;13:385.

7. Ljungström L, Andersson R, Jacobsson G. Incidences of community onset severe sepsis, Sepsis-3 sepsis, and bacteremia in Sweden-a prospective population-based study. PLoS ONE. 2019;14(12):e0225700.

8. Bou-Antoun S, Davies J, Guy R, Johnson AP, Sheridan EA, Hope RJ. Descriptive epidemiology of Escherichia coli bacteraemia in England, April 2012 to March 2014. Euro Surveill. 2016;21(35).

9. Mehl A, Åsvold BO, Lydersen S, Paulsen J, Solligård E, Damås JK, et al. Burden of bloodstream infection in an area of Mid-Norway 2002-2013: a prospective population-based observational study. BMC Infect Dis. 2017:17(1):205

10. Sawatwong P, Sapchookul P, Whistler T, Gregory CJ, Sangwichian O, Makprasert S, et al. High burden of extended-spectrum $\beta$-lactamaseproducing Escherichia coli and Klebsiella pneumoniae bacteremia in older adults: a seven-year study in two rural Thai provinces. Am J Trop Med Hyg. 2019;100(4):943-51.

11. Pitout JD, Laupland KB. Extended-spectrum beta-lactamase-producing Enterobacteriaceae: an emerging public-health concern. Lancet Infect Dis. 2008;8(3):159-66.

12. Laupland KB, Church DL, Vidakovich J, Mucenski M, Pitout JD. Community-onset extended-spectrum beta-lactamase (ESBL) producing Escherichia coli: importance of international travel. J Infect. 2008;57(6):441-8.

13. Laupland KB. Incidence of bloodstream infection: a review of populationbased studies. Clin Microbiol Infect. 2013;19(6):492-500.

14. Laupland KB, Schonheyder HC, Kennedy KJ, Lyytikainen O, Valiquette L, Galbraith J, et al. Rationale for and protocol of a multi-national population-based bacteremia surveillance collaborative. BMC Res Notes. 2009;2:146.

15. Laupland KB, Pasquill K, Parfitt EC, Naidu P, Steele L. Burden of community-onset bloodstream infections, Western Interior, British Columbia, Canada. Epidemiol Infect. 2016;144(11):2440-6.
16. Laupland KB, Church DL. Population-based epidemiology and microbiology of community-onset bloodstream infections. Clin Microbiol Rev. 2014:27(4):647-64.

17. Laupland KB, Lyytikainen O, Sogaard M, Kennedy KJ, Knudsen JD, Ostergaard C, et al. The changing epidemiology of Staphylococcus aureus bloodstream infection: a multinational population-based surveillance study. Clin Microbiol Infect. 2013;19(5):465-71.

18. StataCorp. Stata Statistical Software: Release 15. College Station, TX: StataCorp LLC: 2017.

19. Eurostat. EU 28-country 2018 population on 1 January by age and sex. 2019. Available from: https://appsso.eurostat.ec.europa.eu/nui/show.do? dataset=demo_pjan\&lang=en. Accessed 20 Nov 2019.

20. Dohoo IR, Martin SW, Stryhn H. Methods in epidemiologic research Charlottetown, PEI.: VER, Inc.; 2012.

21. Al-Hasan MN, Lahr BD, Eckel-Passow JE, Baddour LM. Antimicrobial resistance trends of Escherichia coli bloodstream isolates: a population-based study, 1998-2007. J Antimicrob Chemother. 2009;64(1):169-74.

22. de Kraker ME, Wolkewitz M, Davey PG, Koller W, Berger J, Nagler J, et al. Burden of antimicrobial resistance in European hospitals: excess mortality and length of hospital stay associated with bloodstream infections due to Escherichia coli resistant to third-generation cephalosporins. J Antimicrob Chemother. 2011;66(2):398-407.

23. Abernethy JK, Johnson AP, Guy R, Hinton N, Sheridan EA, Hope RJ. Thirty day all-cause mortality in patients with Escherichia coli bacteraemia in England. Clin Microbiol Infect. 2015;21(3):251.e1-8.

24. Anunnatsiri S, Towiwat P, Chaimanee P. Risk factors and clinical outcomes of extended spectrum beta-lactamase (ESBL)-producing Escherichia coli septicemia at Srinagarind University Hospital, Thailand. Southeast Asian J Trop Med Public Health. 2012;43(5):1169-77.

25. Esteve-Palau E, Solande G, Sanchez F, Sorli L, Montero M, Guerri R, et al. Clinical and economic impact of urinary tract infections caused by ESBLproducing Escherichia coli requiring hospitalization: a matched cohort study. J Infect. 2015;71(6):667-74.

26. Uslan DZ, Crane SJ, Steckelberg JM, Cockerill FR 3rd, St Sauver JL, Wilson WR, et al. Age- and sex-associated trends in bloodstream infection: a population-based study in Olmsted County, Minnesota. Arch Intern Med. 2007;167(8):834-9.

27. Skogberg K, Lyytikäinen O, Ruutu P, Ollgren J, Nuorti JP. Increase in bloodstream infections in Finland, 1995-2002. Epidemiol Infect. 2008;136(1):108-14

28. The World Bank. World Bank Country and Lending Groups: Classification. 2018. Available from: https://datahelpdesk.worldbank.org/knowledgeb ase/articles/906519-world-bank-country-and-lending-groups. Accessed 13 Feb 2019.

\section{Publisher's Note}

Springer Nature remains neutral with regard to jurisdictional claims in published maps and institutional affiliations.
Ready to submit your research? Choose BMC and benefit from:

- fast, convenient online submission

- thorough peer review by experienced researchers in your field

- rapid publication on acceptance

- support for research data, including large and complex data types

- gold Open Access which fosters wider collaboration and increased citations

- maximum visibility for your research: over 100M website views per year

At BMC, research is always in progress.

Learn more biomedcentral.com/submissions 